\title{
Activity of Neurons in Monkey Globus Pallidus During Oculomotor Behavior Compared With That in Substantia Nigra Pars Reticulata
}

SooYoon Shin and Marc A. Sommer

J Neurophysiol 103:1874-1887, 2010. First published 27 January 2010; doi:10.1152/jn.00101.2009

You might find this additional info useful...

Supplemental material for this article can be found at:

http://jn.physiology.org/content/suppl/2010/07/07/00101.2009.DC1.html

This article cites 61 articles, 33 of which can be accessed free at:

http://jn.physiology.org/content/103/4/1874.full.html\#ref-list-1

This article has been cited by 1 other HighWire hosted articles

High-frequency, short-latency disinhibition bursting of midbrain dopaminergic neurons

Collin J. Lobb, Charles J. Wilson and Carlos A. Paladini

J Neurophysiol, May , 2011; 105 (5): 2501-2511.

[Abstract] [Full Text] [PDF]

Updated information and services including high resolution figures, can be found at:

http://jn.physiology.org/content/103/4/1874.full.html

Additional material and information about Journal of Neurophysiology can be found at:

http://www.the-aps.org/publications/jn

This information is current as of April 4, 2012.

Journal of Neurophysiology publishes original articles on the function of the nervous system. It is published 12 times a year 


\title{
Activity of Neurons in Monkey Globus Pallidus During Oculomotor Behavior Compared With That in Substantia Nigra Pars Reticulata
}

\author{
SooYoon Shin ${ }^{1}$ and Marc A. Sommer ${ }^{1,2}$ \\ ${ }^{1}$ Department of Neuroscience, Center for the Neural Basis of Cognition and Center for Neuroscience at the University of Pittsburgh, \\ University of Pittsburgh, Pittsburgh, Pennsylvania; and ${ }^{2}$ Department of Biomedical Engineering and Center for Cognitive Neuroscience, \\ Duke University, Durham, North Carolina
}

Submitted 4 February 2009; accepted in final form 25 January 2010

\begin{abstract}
Shin SY, Sommer MA. Activity of neurons in monkey globus pallidus during oculomotor behavior compared with that in substantia nigra pars reticulata. J Neurophysiol 103: 1874-1887, 2010. First published January 27, 2010; doi:10.1152/jn.00101.2009. The basal ganglia are a subcortical assembly of nuclei involved in many aspects of behavior. Three of the nuclei have high firing rates and inhibitory influences: the substantia nigra pars reticulata ( $\mathrm{SNr}$ ), globus pallidus interna (GPi), and globus pallidus externa (GPe). The $\mathrm{SNr}$ contains a wide range of visual, cognitive, and motor signals that have been shown to contribute to saccadic eye movements. Our hypothesis was that GPe and GPi neurons carry similarly diverse signals during saccadic behavior. We recorded from $\mathrm{GPe}, \mathrm{GPi}$, and $\mathrm{SNr}$ neurons in monkeys that made memory-guided saccades and found that neurons in all three structures had increases or decreases in activity synchronized with saccade generation, visual stimulation, or reward. Comparing GPe neurons with GPi neurons, we found relatively more visual-related activity in GPe and more reward-related activity in GPi. Comparing both pallidal samples with the $\mathrm{SNr}$, we found a greater resemblance between GPe and $\mathrm{SNr}$ neurons than that between GPi and $\mathrm{SNr}$ neurons. As expected from a known inhibitory projection from $\mathrm{GPe}$ to $\mathrm{SNr}$, there was a general reversal of sign in activity modulations between the structures: bursts of activity were relatively more common in GPe and pauses more common in $\mathrm{SNr}$. We analyzed the response fields of neurons in all three structures and found relatively narrow and lateralized fields early in trials (during visual and saccadic events) followed by a broadening later in trials (during reward). Our data reinforce an emerging, new consensus that the GPe and $\mathrm{GPi}$, in addition to the $\mathrm{SNr}$, contribute to oculomotor behavior.
\end{abstract}

\section{N T R O D U C T I O N}

The basal ganglia are important for the control of movements (DeLong 1981; Hikosaka and Wurtz 1989; Hikosaka et al. 2000; Mink 1996). Many nuclei comprise the basal ganglia, including the striatum (caudate and putamen), the globus pallidus externa (GPe), the globus pallidus interna (GPi), and the substantia nigra pars reticulata $(\mathrm{SNr})$. The basic layout is that the striatum receives inputs from cerebral cortex, signals are processed through various pathways, and signals exit through the GPi and SNr (Graybiel and Ragsdale Jr 1979; Niijima and Yoshida 1982). A direct pathway from caudate to $\mathrm{SNr}$ contributes to saccadic eye movements (Fig. 1A, blue line at right) (Handel and Glimcher 1999, 2000; Hikosaka and Wurtz 1983a,b,c,d, 1985; Hikosaka et al. 1989a,b, 2000). A second direct pathway runs from putamen to GPi (Fig. $1 A$, blue line at left) and indirect pathways course through the GPe (Fig. 1A, orange lines). The SNr, GPi, and GPe are similar in having

Address for reprint requests and other correspondence: S.Y. Shin, Department of Neuroscience, A210 Langley Hall, University of Pittsburgh, Pittsburgh, PA 15260 (E-mail: sys3@pitt.edu). high spontaneous firing rates and inhibitory influences. We ventured beyond the classic caudate-SNr pathway to systematically examine the activity of GPe and GPi neurons during oculomotor behavior.

Our main goal was to determine whether the firing rates of GPe and GPi neurons are modulated in saccadic tasks and, if they are, to quantitatively characterize the modulation. We predicted that the activity of some neurons in GPi is related to oculomotor behavior because deep brain stimulation and pallidotomy in human GPi are known to affect eye movements (Blekher et al. 2000; Fawcett et al. 2005; O'Sullivan et al. 2003; Straube et al. 1998). In addition to these clinical reports, recent laboratory studies implicate pallidal neurons in oculomotor behavior. Kato and Hikosaka (1995) described saccaderelated activity in GPe during a combined hand-eye task. Pharmacological manipulation of indirect pathways influence saccadic tasks (Nakamura and Hikosaka 2006). Hong and Hikosaka (2008) found that GPi is a source of reward-related signals to the lateral habenula during a reward-biased oculomotor task. A recent study reported that some pallidal neurons have activity around the time of a saccade that is enhanced when monkeys look away from a visual target as opposed to toward a target (Yoshida and Tanaka 2009a).

The previous reports of GPe and GPi neurons analyzed specific types of signals, but there has been no general assessment of the signal content carried by pallidal neurons in oculomotor tasks. Nor has there been any direct comparison of pallidal neurons with SNr neurons. We hypothesized that the activity of neurons in GPe and GPi exhibit multiple types of activity during oculomotor behavior. As found previously in the $\mathrm{SNr}$, this activity could represent a spectrum of events such as detection of a visual stimulus, memory of it during a delay period, saccade generation toward it (Handel and Glimcher 2000; Hikosaka and Wurtz 1983a,c), and delivery of reward. We recorded from GPe and GPi neurons, mapped their response fields, and analyzed their patterns of activity while monkeys made memory-guided saccades to the response field centers. In contrast to previous methods that used fixed-target eccentricities (e.g., Hong and Hikosaka 2008; Kato and Hikosaka 1995; Yoshida and Tanaka 2009a), the approach that we adopted-tailoring the target location to each neuron's response field center-is more time consuming but should optimize the analysis of neuronal signals. We recorded from $\mathrm{SNr}$ neurons in the same monkeys using the same tasks. The results provide the first quantitative assessment of the diversity and spatial representations of visual, delay, saccade, and rewardrelated signals in $\mathrm{GPe}$ and $\mathrm{GPi}$, in the context of direct comparisons with $\mathrm{SNr}$. 
A

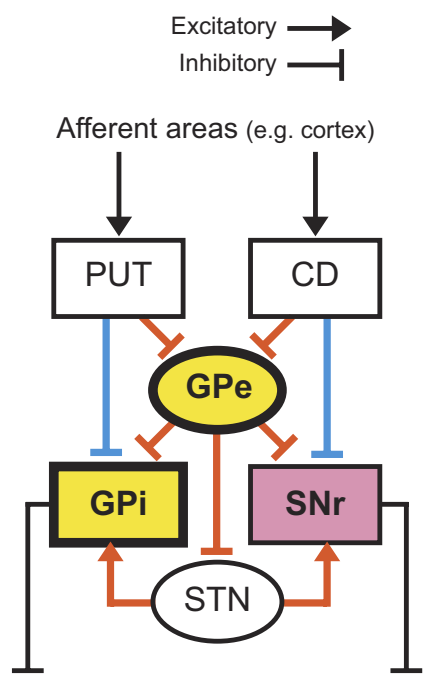

Efferent areas (e.g. thalamus, SC)

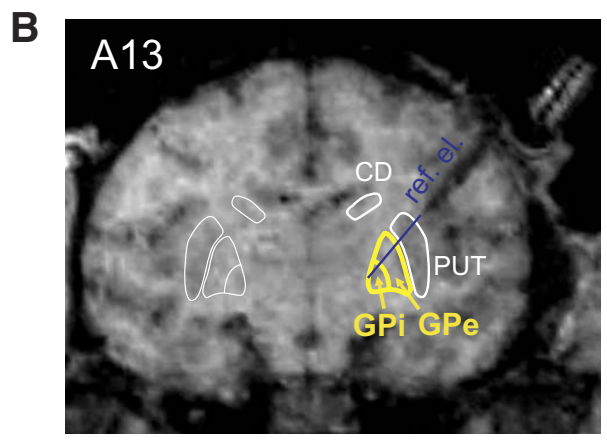

C

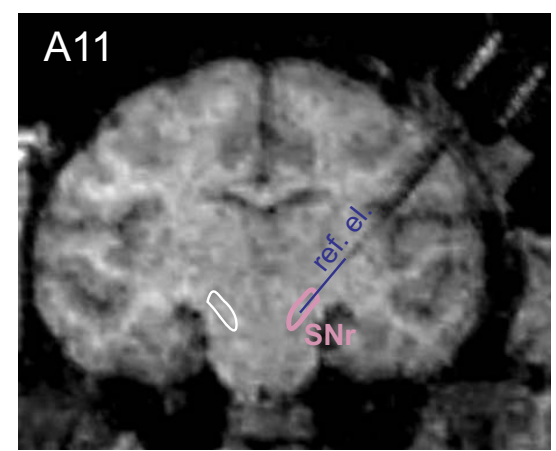

FIG. 1. The basal ganglia. A: diagram of basic circuits: blue lines, direct pathways; orange lines, indirect pathways. PUT, putamen; $\mathrm{CD}$, caudate nucleus; STN, subthalamic nucleus; other abbreviations as in text. $B$ : coronal magnetic resonance imaging (MRI) from one monkey showing our electrode approach in relation to the basal ganglia. The reference electrode (ref. el.) was implanted above the location of task-related pallidal neurons, but we kept it out of the putamen to preclude any risk to the basal ganglia during imaging. The track that the electrode would have taken in a recording experiment is shown with the blue line. $C$ : similarly, our electrode approach in relation to the substantia nigra pars reticulata $(\mathrm{SNr})$, showing a second, simultaneously implanted ref. el. Note that the ref. el. location in $B$ was selected to be near the medial edge of the pallidal recording range, so that its image could be distinguished clearly from the other ref. el. pointing at $\mathrm{SNr}$ only 2 $\mathrm{mm}$ posterior to it (A13 and A11 indicate the stereotaxic planes of the MRI slices). Exact boundaries of basal ganglia nuclei could not always be seen in the MRI sections, but were determined by comparing the images with stereotaxic atlases.
METHOD S

\section{Surgical procedure}

Three rhesus monkeys (Macaca mulatta) were surgically prepared in aseptic conditions under isofluorothane anesthesia. We implanted scleral search coils for eye position monitoring (Judge et al. 1980), a chamber for neuronal recordings, and a post for immobilizing the head during experiments (see Sommer and Wurtz 2000 for details). Dental acrylic held together the chamber, head post, and eye coil plugs and the entire implant was attached to the skull via bone screws. The chamber was centered at 11 anteroposterior (AP), 28 mediolateral (ML), and angled $40^{\circ}$ from vertical in the coronal plane to access the $\mathrm{GPe}, \mathrm{GPi}$, and $\mathrm{SNr}$ in the right hemisphere. We assessed chamber placement using magnetic resonance imaging (MRI) with reference electrodes (Fig. 1, $B$ and $C$ ). MRI images were compared with stereotaxic atlases to confirm the location of the basal ganglia structures (Martin et al. 1997; Paxinos et al. 2000; http://www.brainmaps. org). All procedures were approved by the Institute for Animal Care and Use Committee in accordance with the National Institutes of Health Guide for the Care and Use of Animals.

\section{Behavioral paradigm}

First, we determined the preferred direction of a neuron by having the monkey make memory-guided saccades as described in the following text to targets in eight different directions (the cardinal axes and diagonals). For this assessment we used an amplitude estimated to be optimal for the neuron. From inspection of on-line spike rasters and spike density functions, we determined the direction that elicited the highest firing rate of the neuron. If two neighboring directions yielded similar maximal firing rates, the best direction was considered to be the angle that bisected those two directions; thus our resolution was $22.5^{\circ}$ in circular angle. Along this best direction, we honed in on the optimal amplitude by having the monkey make visually guided saccades to targets at multiple eccentricities (typically eight: $2,5,10$, $30,40,50$, and $60^{\circ}$; if pressed for time we used 5,10 , and $30^{\circ}$ ). If the best amplitude determined by this procedure differed from the initially estimated best amplitude, we reran the directional tuning assessment using the newly determined amplitude. See Sommer and Wurtz (2004) for more details of this iterative procedure. By alternating between these direction and amplitude tests and fine-tuning the target locations accordingly, we were able to find the "hot spot" of the neuron's response field in a time-efficient manner.

After determining a neuron's best direction and amplitude, we ran the monkey on a memory-guided saccade task with two targets, one at the response field center and one at the diametrically opposite location. We typically collected 10-20 trials at each location, but sometimes up to 40 trials if a neuron's isolation was exceptionally stable. Trials in the diametrically opposite location were run (but not analyzed) to maintain spatial symmetry, thus discouraging the monkey from making anticipatory saccades and precluding the possibility of causing inadvertent biases to the natural response field lateralities. We focused on the memory-guided saccade task because it permits temporal separation of visual-, delay-, and saccade-related activity (Hikosaka and Wurtz 1983c; Mays and Sparks 1980) and it was used in many previous studies of the SNr (e.g., Basso and Liu 2007; Handel and Glimcher 2000; Hikosaka and Wurtz 1983c). A monkey fixated a spot for a random duration of 500-800 ms, a target appeared in the center of the response field for $50 \mathrm{~ms}$, a delay period of $500-1,000 \mathrm{~ms}$ ensued, and then the fixation spot disappeared, which was the cue to move. After making the saccade to the location of the (absent) target, the monkey had to fixate the target location for $200 \mathrm{~ms}$. Then the target reappeared and $300 \mathrm{~ms}$ later, a reward was delivered. The electronic window for verifying fixations on-line was $3^{\circ}$ square. The window around the target location for verifying a correct saccade on-line was adjusted by the investigator as a function of eccentricity (larger windows for larger eccentricities). For $15^{\circ}$ eccentricities, as an example, the window was typically $4^{\circ}$ horizontal $\times 6^{\circ}$ vertical. It was larger in the vertical dimension because of normal upshifts in making memoryguided saccades (White et al. 1994). Regardless of these on-line windows, we always inspected every trial off-line and omitted trials from analysis if a fixation or saccade endpoint fell outside the main cluster of eye positions.

\section{Recording procedure}

GPe and GPi neurons were identified by their anatomical location relative to landmarks such as the putamen, internal capsule, lateral 
geniculate nucleus, and third cranial (oculomotor) nerve. Physiological properties of the neurons such as action potential shapes and streaks of intermittent pauses that are common in GPe provided further confirmation (DeLong 1971). We sometimes encountered both GPe and GPi neurons on single penetrations (Fig. 1B). Other days, we encountered a few millimeters of the GPe but could not enter GPi because we were near the caudal boundary of GPe (and GPe is broader than GPi). The top of GPe was easily identifiable due to the sudden increase in spontaneous firing rate at the putamen/GPe border (DeLong 1971). The GPe/GPi border was not always obvious because neurons in both structures have high firing rates and there is only a thin laminar border between them. Therefore after recording all of the neurons, but before quantitatively analyzing their signals, we applied a stringent depth criterion: for a neuron to be classified as GPi it had to be located $>2.5-3 \mathrm{~mm}$ below the top of GPe. According to atlases (Martin et al. 1996; Paxinos et al. 2000) and previous studies (Anderson and Turner 1991; Elias et al. 2007), only GPi neurons are found that deep. The criterion varied from 2.5 to $3 \mathrm{~mm}$ due to gradual changes in the GPe/GPi border with AP location. Task-modulated neurons were clustered within a volume of a few cubic millimeters, accessible by three to four adjacent grid holes each separated by $1 \mathrm{~mm}$ (Crist Instruments, Hagerstown, MD), and were found at the posterior and mediodorsal portion of GPe and GPi based on the landmarks noted earlier and MRI. The distribution of recording depths of GPe and GPi neurons is shown in Supplemental Fig. S1A. ${ }^{1}$ As a post hoc test of the GPe/GPi categorization, after analyzing all of the neuronal signals we examined how they varied with depth through the pallidum (Supplemental Fig. S1B, using the Reward-Visual Index analysis described in the following text). We found a sudden change in signal content at the estimated GPe/GPi boundary, implying that few, if any, of the GPe or GPi neurons were misclassified.

We recorded from the $\mathrm{SNr}$ from the same chamber in all three monkeys (Fig. 1C). Finding the SNr allowed us to be certain that we were not accidentally including SNr neurons in our pallidal samples and recording from its neurons provided a firm basis of comparison with pallidal neurons (rather than just comparing our pallidal neurons with prior descriptions of $\mathrm{SNr}$ neurons in the literature). $\mathrm{SNr}$ neurons were identified by their high baseline firing rate (50-90 spikes/s) (Hikosaka and Wurtz 1983a) and their proximity to nearby landmarks, including the internal capsule, subthalamic nucleus, zona incerta, oculomotor nucleus, and rostral interstitial nucleus of the medial longitudinal fasciculus. Relative to the GPi, the $\mathrm{SNr}$ was $4-5 \mathrm{~mm}$ more posterior, about $4 \mathrm{~mm}$ deeper, and on the other side of (medial to) the easily identifiable internal capsule.

In all structures, the activity of single neurons was recorded extracellularly with parylene-insulated tungsten electrodes (FHC, Bowdoinham, ME). Action potentials were amplified and isolated using time and amplitude criteria on a digital oscilloscope. Time stamps of action potentials were stored in data files. Visual stimuli were back-projected using an LCD projector onto a tangent screen 58 $\mathrm{cm}$ in front of the monkey. Eye-position data were collected at 1-ms resolution. Data collection and the behavioral paradigm were under the control of the REX real-time system (Hays et al. 1982).

\section{Data analysis}

To determine the signals carried by the neurons, we measured average firing rates in five epochs of the memory-guided saccade task: a baseline epoch 0 to $100 \mathrm{~ms}$ before target onset, a visual epoch 50 to $150 \mathrm{~ms}$ after target onset, a delay epoch 0 to $100 \mathrm{~ms}$ before the cue to move, a saccade epoch $50 \mathrm{~ms}$ before to $50 \mathrm{~ms}$ after saccade initiation, and a reward epoch 0 to $100 \mathrm{~ms}$ before reward onset. We ran an ANOVA on the firing rates across the five epochs and, if this was significant at $P<0.01$, the neuron was considered to be taskmodulated. We used a parametric ANOVA if the firing rate distribu-

\footnotetext{
${ }^{1}$ The online version of this article contains supplemental data.
}

tions passed a test of normality and equal variance, or a nonparametric one otherwise (Kruskal-Wallis one-way ANOVA on ranks). For neurons with significant ANOVA results, we performed an all-pairwise multiple-comparison test (Student-Newman-Keuls or Dunn's at $P<$ $0.05)$ to compare average firing rates between the various epochs. A neuron had a visual-related signal if its firing rate in the visual epoch differed from that in the baseline epoch, a delay-related signal if its firing rate in the delay epoch differed from that in the baseline epoch, a saccade-related signal if its firing rate in the saccade epoch differed from that in both the delay and baseline epochs, and a reward-related signal if the firing rate in the reward epoch differed from that in both the baseline and saccadic epochs. The test for saccade-related signals involved a double comparison (vs. both the delay and baseline epochs) to make sure that apparent saccadic activity was neither an extension of delay activity nor a simple return to baseline activity. The analyses typically involved 10 trials, but ranged from a minimum of 7 to a maximum of 40 trials.

On a closed-circuit infrared camera system we watched the monkeys carefully for other types of body movements that might modulate neuronal activity, such as orofacial and limb movements. The animals made occasional postural adjustments, limb movements, and facial movements such as blinking and licking. If we determined that a neuron was modulated by such movements we excluded it from further analyses. We routinely gave the animals free drops of liquid reward to see whether this evoked licking, somatosensory, or other nontask-related modulations in activity, and excluded such neurons, but we were not set up to quantitatively assess such fine details. Thus although we could detect reward-related signals, and we think that most were not due to trivial reasons such as licking, we remain cautious in interpreting them.

We analyzed how signals varied as a function of depth through the pallidum using an index that quantified the relative amounts of visualand reward-related activity in single neurons. The ratio of these activity types, as described in RESULTS, was the main difference in signal content between GPe and GPi. The Reward-Visual Index (RVI) was the contrast ratio of the visual and reward activity, with each activity level measured relative to baseline and absolute-valued to treat increases and decreases identically: RVI $=(\mid$ reward-baseline $|-|$ visual-baseline $\mid) /(\mid$ rewardbaseline $|+|$ visual-baseline $\mid)$. If RVI $=0$, a neuron had equivalent reward and visual modulations; if RVI was between 0 and 1 , a neuron had stronger reward than visual modulations; and if RVI was between 0 and -1 , a neuron had stronger visual than reward modulations.

We determined the spatial tuning curves of neurons for which we obtained memory-guided task data in all eight directions at the optimal amplitude. We routinely collected 10 trials of data at each target direction. Since the major signals of neurons were visual-, saccade-, and reward-related (delay-related activity was relatively rare), we focused on those three signals. They were measured as average firing rates in the epochs described earlier. First, to determine whether the signals varied as a function of direction, for each signal type we ran an ANOVA on firing rates in the eight directions $(P<$ 0.05 significance level). An insignificant ANOVA suggested that the neuron was omnidirectionally tuned. To characterize the tuning at a finer scale that considered modulations at each direction individually, we also compared the firing rates with baseline activity on a directionby-direction basis. The baseline epoch preceded target onset and therefore was not influenced by target direction; thus when analyzing spatial tuning plots we used a single baseline firing rate derived by averaging baseline data from all eight directions. We constructed separate polar plots of spatial tuning for each type of signal conveyed by a neuron. Relative to baseline, a neuron could have a significant change in activity in one or more (sometimes all) directions ( $t$-test, $P<0.05$, Bonferroni corrected for multiple comparisons against the baseline activity). Small numbers of significant directions indicated sharp tuning, large numbers broad tuning.

In addition to analyzing spatial characteristics of the signals we also analyzed their timing, specifically the point at which they started during 
a trial. We determined onset latencies of each signal type in the following manner (see Sommer and Wurtz 2004 for details). First, we generated spike density functions $(\sigma=10 \mathrm{~ms})$ aligned to events of interest such as visual stimulus onset. The mean and SD of the baseline activity provided the threshold for determining the time at which event-related modulations became significant (threshold was twice the SD of baseline). This crossing time was the onset latency. If the neuronal signal was an increase in activity, the firing rate had to exceed threshold (2SDs above baseline); if the signal was a pause in activity, the firing rate had to drop below threshold (2SDs below baseline).

\section{R E S U L T S}

\section{Task-related signals carried by pallidal neurons}

We recorded from 320 GPe neurons in three monkeys and 101 GPi neurons in two monkeys. Overall, 34\% of the neurons ( $n=143$ ) were modulated significantly in the memory-guided saccade task ( $38 \%$ of GPe neurons, $n=121 ; 22 \%$ of GPi neurons, $n=22$ ).

Examples of single-neuron activity in GPe and GPi during the memory-guided saccade task are shown in Figs. 2 and 3, respectively. We found a wide variety of activity patterns. The neurons typically had high spontaneous activity (means and SEs: $78.9 \pm 28.6$ spikes/s for GPe; $76.3 \pm 23.2$ spikes/s for

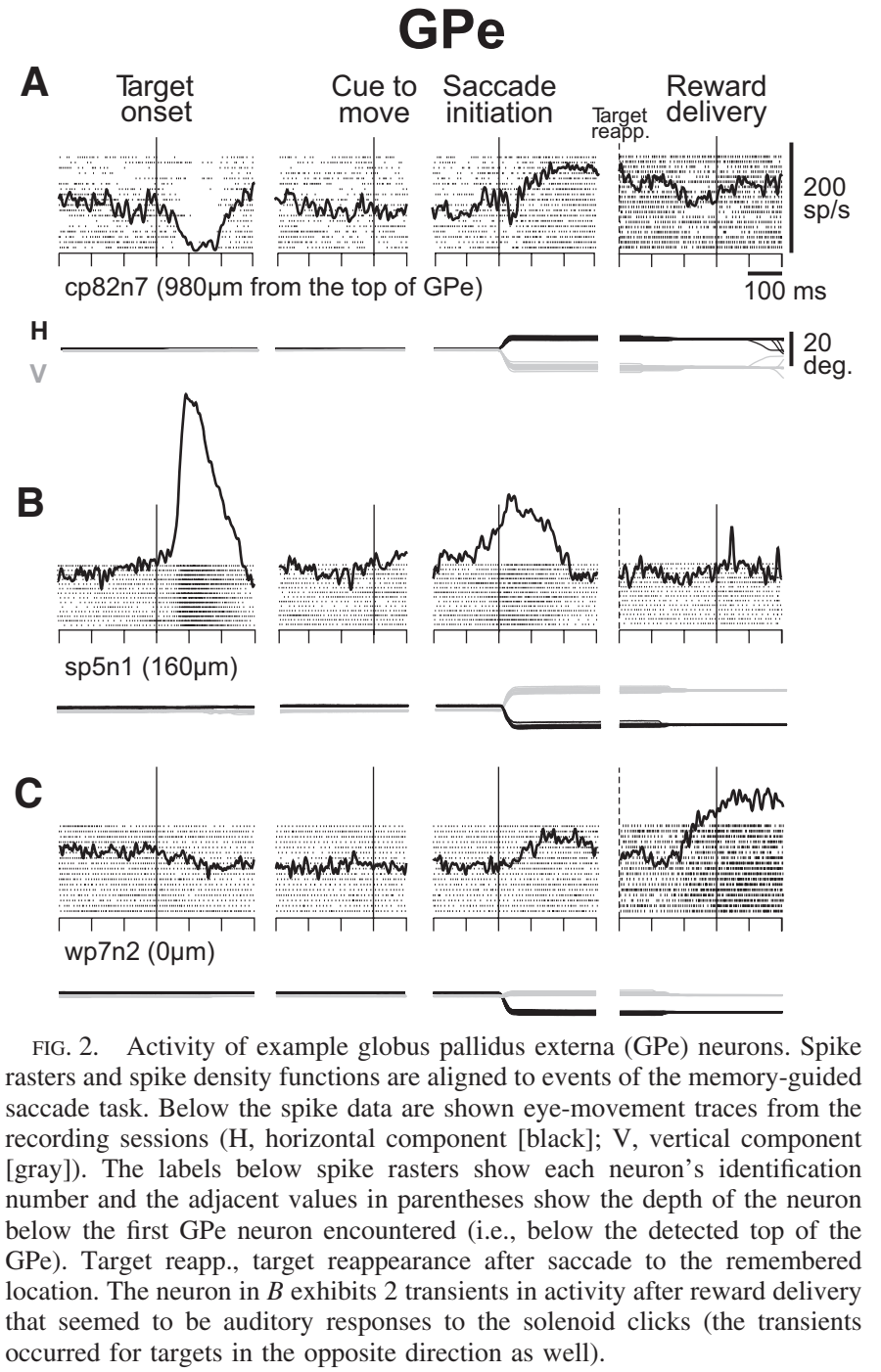

\section{GPi}

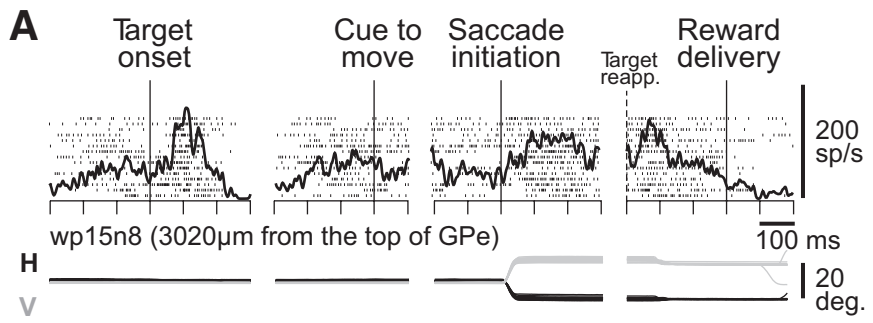

B
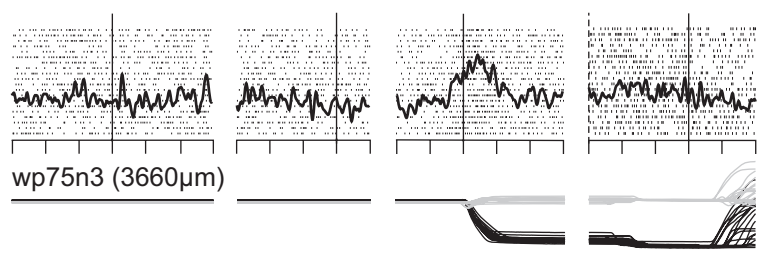

C
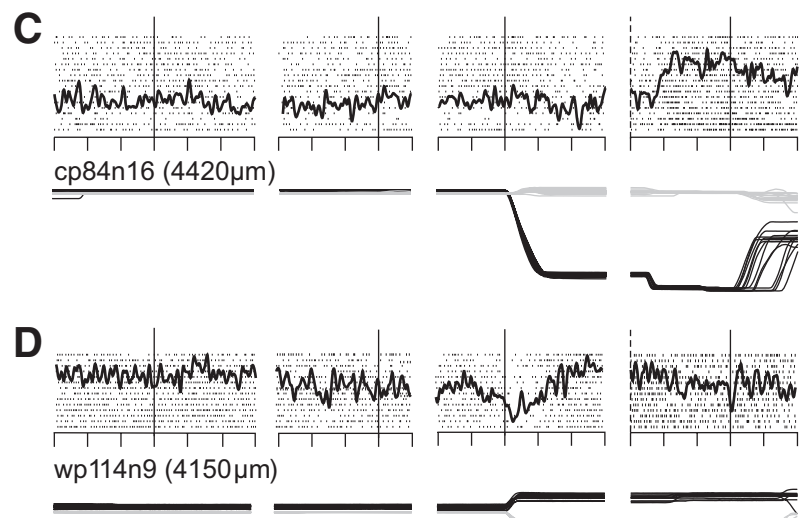

FIG. 3. Activity of example globus pallidus interna (GPi) neurons. All conventions are the same as in Fig. 2. A shows the only visual-related neuron we found in GPi. In that same panel, a transient visual response to target reapp. can be seen; such responses were rare and brief. They ended (as did modulations seemingly linked to saccade termination) prior to the reward analysis epoch $100-0$ ms before reward delivery.

GPi). Relative to these baseline firing rates, GPe and GPi neurons could show increases or decreases in activity in association with events such as visual stimulation (Figs. 2, $A$ and $B$ and $3 A$ ), delay (Figs. $2 C$ and $3 D$ ), saccade generation (Figs. $2 B$ and $3, B$ and $D$ ), and reward (Figs. $2 C$ and $3 C$ ). As can be seen in Figs. 2 and 3, single neurons could carry one signal or any combination of signals (quantified in the following text). Aside from these signals of interest, there were rare, miscellaneous signals that we did not quantify such as phasic visual responses to target reappearance (Fig. $3 A$ ) and apparent auditory responses to reward solenoid clicks (Fig. 2B).

Due to the wide variety of signals, pooling the data to show population firing rates tended to obscure the modulations (Supplemental Fig. S2A). It was evident, however, that for both GPe and GPi and for each type of signal, the average increasing and decreasing modulations were similar (Supplemental Fig. S2, $B$ and $C$ ). We analyzed the timings of the task-related modulations quantitatively (see METHODS) and found that GPe neurons had remarkably quick visual latencies regardless of whether the responses were of the increasing or decreasing type (means 50 and $51 \mathrm{~ms}$, respectively; Table 1). The one visual-related GPi neuron that we found was comparable (see Fig. 3A). In general across all of the samples, saccade-related 
TABLE 1. Distribution of signal latencies

\begin{tabular}{|c|c|c|c|c|c|c|}
\hline & \multicolumn{2}{|c|}{ Visual } & \multicolumn{2}{|c|}{ Saccade } & \multicolumn{2}{|c|}{ Reward } \\
\hline & + & - & + & - & + & - \\
\hline GPe & $50 \pm 22$ & $51 \pm 27$ & $-94 \pm 60$ & $-89 \pm 56$ & $-198 \pm 76$ & $-216 \pm 73$ \\
\hline GPi & N/A & N/A & $-75 \pm 34$ & N/A & $-200 \pm 76$ & $-152 \pm 65$ \\
\hline
\end{tabular}

All entries are in units of milliseconds $(\mathrm{ms})$ and represent the time (mean \pm $\mathrm{SD}$ ), when modulation became significant relative to visual target onset (Visual data), saccade initiation (Saccade data), and reward delivery (Reward data), for increasing types of neurons $(+)$ and decreasing types $(-)$. Negative values are times before an event; positive values are after an event.

activity started to modulate around 70-100 ms before saccade onset and reward-related activity around $200 \mathrm{~ms}$ before reward delivery. We found no significant differences in latency ( $t$-test, $P>0.05)$ as a function of area (e.g., GPe saccade vs. GPi saccade) or valence of activity (e.g., GPe saccade increase vs. GPe saccade decrease).

Our quantitative analysis of the full diversity of pallidal signals is summarized in Fig. 4. The overall distributions of signal types were significantly different between the GPe and GPi samples (Fig. 4A; $4 \times 2$ Exact Contingency Table test, $P<0.001)$. Visual responses were far more common in GPe (44\%, or 53/121 task-related neurons) than in GPi $(5 \%, 1 / 22$ task-related neurons). In contrast, reward-related signals were more common in GPi than in GPe (73 vs. 53\% of task-related neurons). Saccade-related signals were found in about a quarter of task-related neurons in both structures $(26 \%$, GPe; $27 \%$, GPi). Delay-related signals were relatively infrequent $(21 \%$, GPe; $18 \%$, GPi).

Individual neurons could carry one signal or multiple signals (Fig. 4B). As classified by the combination of signals they carried, the distributions of neuron types were significantly different in GPe versus GPi $(4 \times 2$ Exact Contingency Table test, $P<0.001)$. GPe neurons were much more likely than GPi neurons to have visual activity as their only signal. Conversely, although reward-related activity was prominent in both structures, GPi neurons were much more likely than GPe neurons to have reward activity as their only signal. The GPi had slightly more saccade-only neurons than GPe and, in both structures, neurons with only delay signals were rare. The percentage of neurons carrying multiple signals was comparable in the two structures.

The various signals carried by the neurons, including the myriad combinations that could be found in single neurons, are illustrated in detail using Venn diagrams in Fig. 4C. Although the Venn diagrams are intricate for the sake of reporting all of the signal combinations, the take-home message is simple. There were three prominent types of neurons: visual-only GPe neurons and reward-only GPe and GPi neurons. Beyond that, the cooccurrence of signals was fairly homogeneously distributed in GPe (the data were too sparse to draw a similar conclusion for GPi).

\section{Comparison with SNr neurons}

We recorded from $\mathrm{SNr}$ neurons in all three monkeys, to confirm that we were not accidentally including SNr neurons in our pallidal samples and to compare task-related properties between pallidal and $\mathrm{SNr}$ neurons. In each monkey, we found that the $\mathrm{SNr}$ was located 5-6 $\mathrm{mm}$ ventroposterior to, and across the distinctive internal capsule from, the GPe and GPi recording sites. Thus we are certain that no $\mathrm{SNr}$ neurons were in the GPe or GPi samples. The SNr neurons had high baseline firing rates, nearly always $>50$ spikes/s, which demonstrated that we were not in the nearby subthalamic nucleus with its baseline firing rates of about 25-30 spikes/s (Hikosaka and Wurtz 1983a; Soares et al. 2004).

As expected from prior work (e.g., Hikosaka and Wurtz $1983 \mathrm{c})$, we found that many $\mathrm{SNr}$ neurons $(45 \%, 42 / 93)$ were modulated in the memory-guided saccade task. Similar to GPe and GPi neurons, the SNr neurons could exhibit either taskrelated bursts or pauses (Fig. 5). At the population level (Fig. 6), the $\mathrm{SNr}$ neurons were more similar to GPe neurons than GPi neurons, in that the majority of modulated $\mathrm{SNr}$ neurons carried visual-related and reward-related signals. Still, the distributions of signal types were different between the two structures $(4 \times$ 2 Exact Contingency Table test, $P=0.002)$. This difference seemed due to the $\mathrm{SNr}$ having more visual activity (69 vs. $44 \%$ ) and less reward activity (29 vs. 53\%) than the GPe. Differences in signal content were even greater between $\mathrm{SNr}$ and GPi $(4 \times 2$ Exact Contingency Table test, $P<0.001)$ because the GPi had such a paucity of visual responses and abundance of reward-related activity, as discussed earlier.

Our finding that SNr neurons are more similar to GPe than GPi neurons is consistent with anatomy, in that the $\mathrm{SNr}$ receives projections from GPe but is relatively isolated from GPi (recall Fig. 1A). The inhibitory nature of the GPe-SNr projection leads to a hypothesis that there may be a sign reversal in signals from GPe to SNr. To test this, we compared the total numbers of increasing versus decreasing signal types in GPe (Fig. 4A, left) and $\mathrm{SNr}$ (Fig. 6A). We found that increasing modulations were more common in GPe $(68 \%$ of its signals) than in $\mathrm{SNr}$ (33\% of its signals). In turn this meant that decreasing modulations were more common in $\mathrm{SNr}$ than in GPe (67 vs. $32 \%$ of signals, respectively). These differences were significantly different (chi-square test, $P<0.0001$ ), supporting the hypothesis that excitatory signals in GPe may contribute, via the known inhibitory projection, to pauses in the $\mathrm{SNr}$.

\section{Tuning of response fields}

Next we analyzed the ranges of target directions and amplitudes that could evoke task-related modulations, i.e., the response fields of the neurons. As described in METHODS, our iterative mapping procedure determined the "hot spot"-optimal direction and amplitude-for each neuron individually. Overall (GPe and GPi neurons pooled), we found that the amplitudes of the response field centers ranged from 2 to $64^{\circ}$ with an average of $18^{\circ}$ and SD of $12^{\circ}$. These amplitudes were comparable in GPe (range: 5-64 $4^{\circ}$ mean: $18^{\circ}$; SD: $11^{\circ}$ ) and GPi (range: $2-60^{\circ}$; mean: $20^{\circ}$; SD: $13^{\circ}$ ). Using the optimal amplitude for each neuron, we evaluated the directional tuning using a set of eight directions (cardinal axes and diagonals). We found that the response field shapes were biased toward one hemifield-i.e., lateralized-in the majority of neurons (77\% of task-modulated GPe neurons, 95/123; 52\% of taskmodulated GPi neurons, 11/21). For those neurons, contralateral response fields were the most common (75\% of lateralized fields for GPe; $64 \%$ for GPi). For $15 \%$ of task-modulated GPe 
$\mathrm{GPe}$

A Signal Type

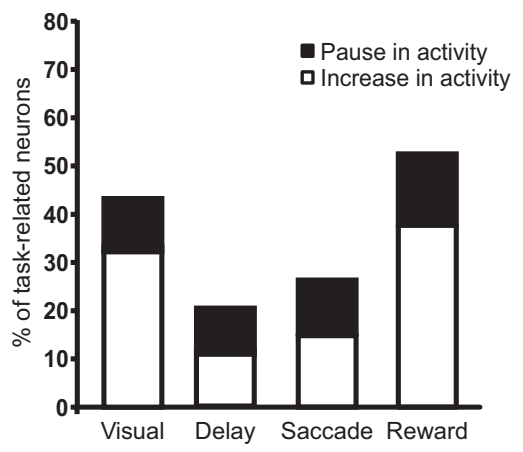

\section{B Neuron Type}
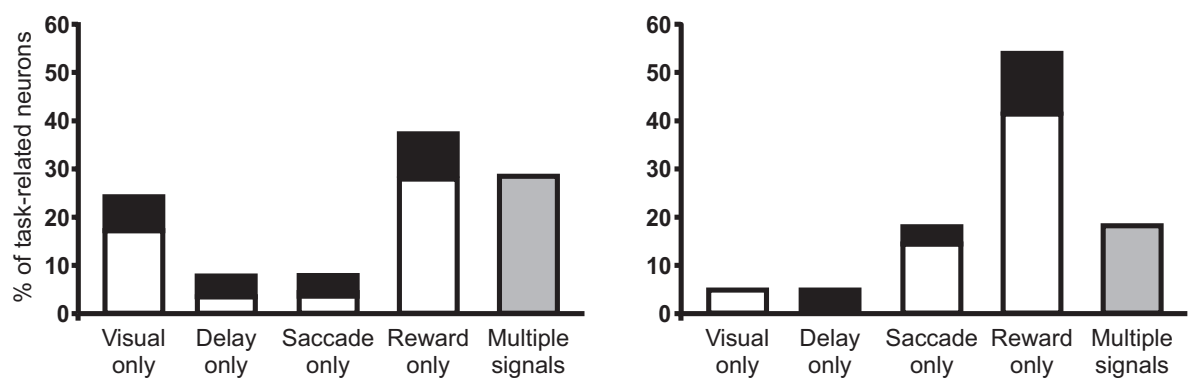

\section{Combination of Signal}
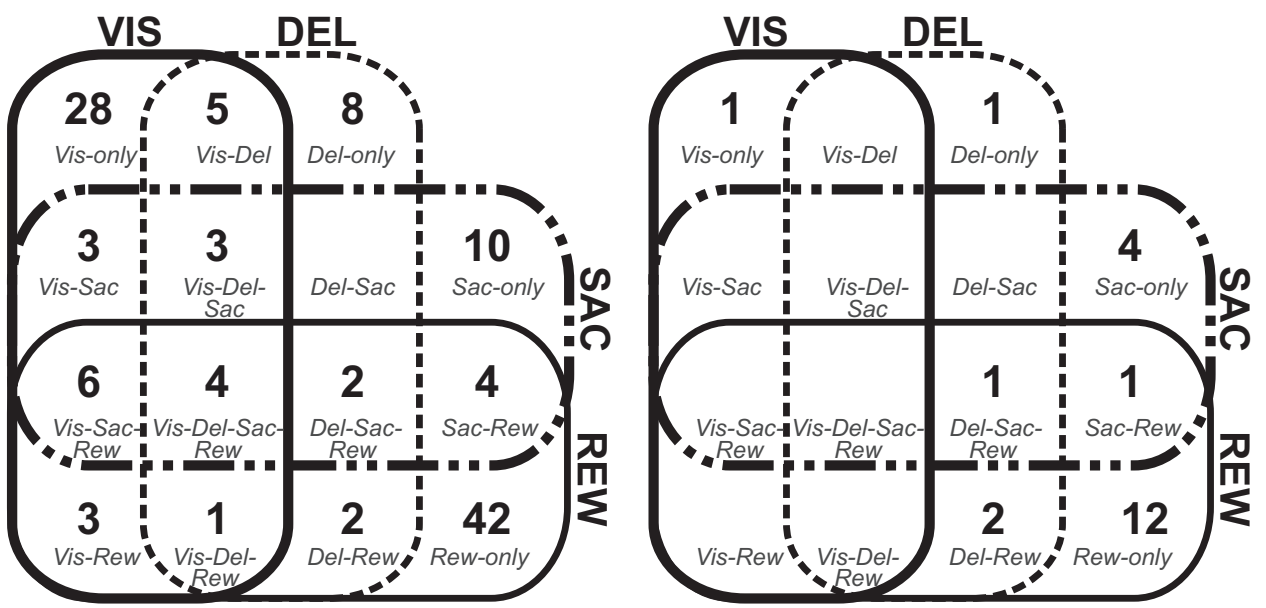

FIG. 4. Population data for GPe (left) and GPi (right). A: distributions of signals carried by task-modulated neurons. Signals were conveyed as increases (white) or decreases (black) relative to the baseline firing rate. $B$ : distributions of neurons categorized by whether they carried one type of signal or multiple types (gray). $C$ : Venn diagrams showing the numbers of neurons that had various combinations of signal types. The 4 categories of signals (VIS, visual; DEL, delay; SAC, saccade; REW, reward) are represented with rounded rectangles using 4 different types of line to help with clarity. Regions overlapped by more than one rectangle represent neurons with the depicted combination of more than one signal. Regions are mutually exclusive (i.e., sum of numbers in all regions is the total number of modulated neurons). Each possible combination of signal is written in italic in the respective regions. and $43 \%$ of task-modulated GPi neurons, the response field was not lateralized but extended into both the contralateral and ipsilateral fields (i.e., bilateral). The remaining neurons had response fields aligned vertically ( $8 \%$ of GPe and $5 \%$ GPi neurons).

For the GPe and GPi task-modulated neurons that were fully tested on memory-guided saccades in eight directions (at each neuron's optimal amplitude), we analyzed the details of receptive field directional tuning. Figure 7 shows example tuning curves (means \pm SEs) of neurons in GPe and GPi and, for comparison, SNr. The rows show data from each anatomical structure and the columns show examples of the tuning for each signal type (the data in each panel were selected to show typical response fields and were not necessarily from the same neuron). In all tuning plots, baseline activity of the neuron is shown with gray curves. As reported earlier, signal modulations could be above or below baseline. Sharpness of tuning varied from neuron to neuron. Some neurons showed fairly sharp tuning with significant modulations for only one to three directions (e.g., the GPe Visual plot). Others had broad or even omnidirectional tuning spanning all eight directions (e.g., the GPe Reward plot). The significantly modulated directions were generally contiguous; in our entire experiment, we found only two neurons with distinctly separated lobes pointing in different directions.

The GPe neurons provided the largest data set with regard to tuning curves $(n=89)$ and they carried all possible signal types, so we concentrated on them for analyzing population tuning data (Fig. 8). The top row (Fig. 8, A-C) shows averaged tuning curves for GPe neurons with increasing types of signals; the 


\section{$\mathrm{SNr}$}

A

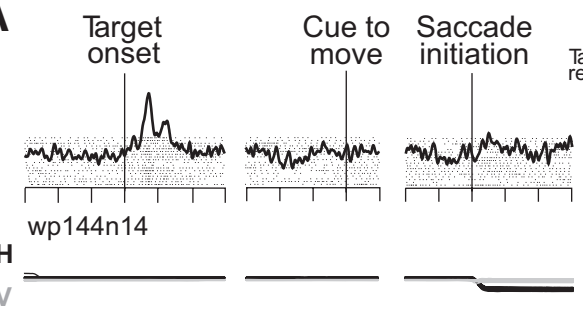

Reward
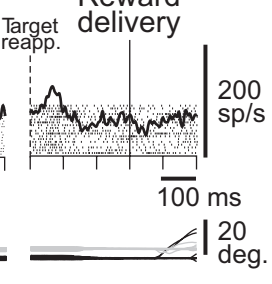

B

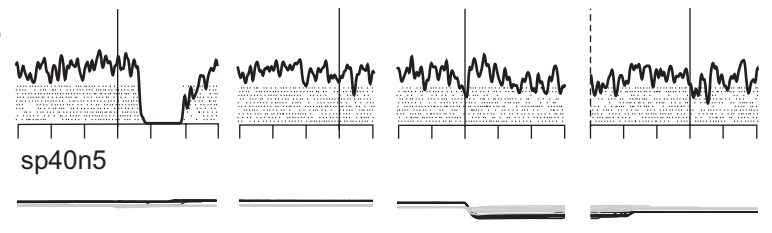

C

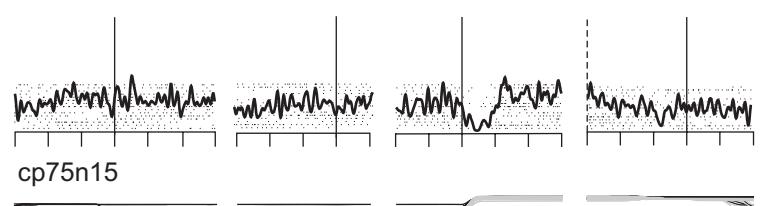

FIG. 5. Activity of example $\mathrm{SNr}$ neurons. Neurons with a visual increase $(A)$, a visual decrease $(B)$, or a saccadic decrease $(C)$ are shown. Other conventions are the same as those in Fig. 2.

bottom row (Fig. 8, $D-F$ ) shows curves for the neurons with decreasing types of signals. Before we constructed the average plots, we normalized the firing rates for each neuron relative to its baseline (thus the average baseline curves, gray, are equal to one). We did not perform any other normalizations; specifically, the directions are veridical (as in Fig. 7, contralateral is to the left and ipsilateral to the right). Visual, saccadic, and reward response fields are separated by columns. We found that the increasing visual responses of GPe neurons were significantly biased contralaterally on average (ANOVA, $P<$ 0.001 , Fig. $8 A$ ). This was also true for most of the individual neurons $(30 / 33,91 \%)$. Most neurons with decreasing visual responses also showed a contralateralized bias $(8 / 9,89 \%)$. In the average tuning curve of those neurons (Fig. $8 D$ ) this bias was not significant by ANOVA $(P>0.05)$, but it was significant in two contralateral directions by $t$-test (asterisks).

In each panel of Fig. 8, the top right insets illustrate the individual tuning vectors for each neuron that contributed to the average plot. The bottom right insets show the number of significant directions for the individual neurons. Most of the GPe visual neurons (increasing and decreasing types, Fig. 8, A and $D$ ) had relatively sharp tuning, with up to three directions that were different from baseline, and none had omnidirectional tuning (i.e., eight modulated directions).

Compared with those visual signals, the average tuning of saccadic signals for both increasing and decreasing types (Fig. 8, $B$ and $E$ ) showed no significance by ANOVA and weaker contralateral biases according to the direction-by-direction $t$ test (Fig. 8, $B$ and $E$, asterisks). At the individual neuron level, significant lateralizations were found in $10 / 15$ neurons $(67 \%)$ with increasing signals and in $3 / 8$ neurons (38\%) with decreasing signals. There was more of a tendency for broad tuning in saccadic signals than that seen for visual signals (bottom insets); a couple of neurons even had omnidirectional saccadic tuning.

Reward-related signals (Fig. 8, $C$ and $F$ ) were even more uniformly tuned across space. The average tuning plots were not significantly biased (by ANOVA), but activity in all eight directions was significantly greater than (Fig. $8 C$ ) or less than (Fig. $8 F$ ) baseline activity (by $t$-test). The population omnidirectionality was due in part to tunings that could point in any direction for individual neurons (top right insets), but even those individual neurons had broad tunings with many occurrences of omnidirectionality (bottom right insets). Only a few individual neurons showed a significant directional bias (in-

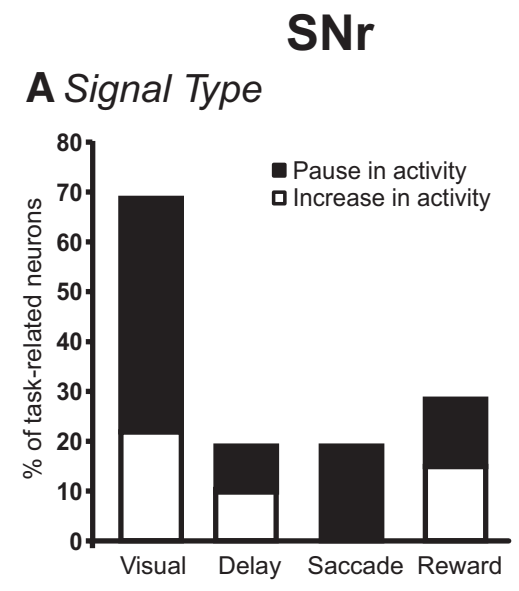

\section{B Neuron Type}

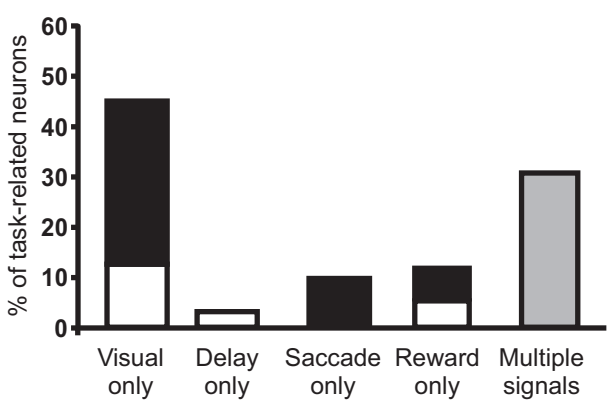

\section{Combination of Signal}

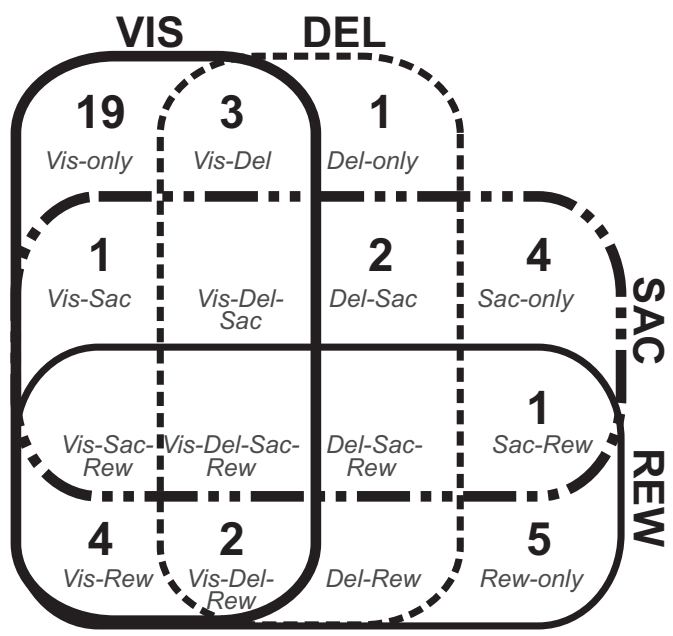

FIG. 6. Population data for signal types in SNr. Conventions are the same as those in Fig. 4. 

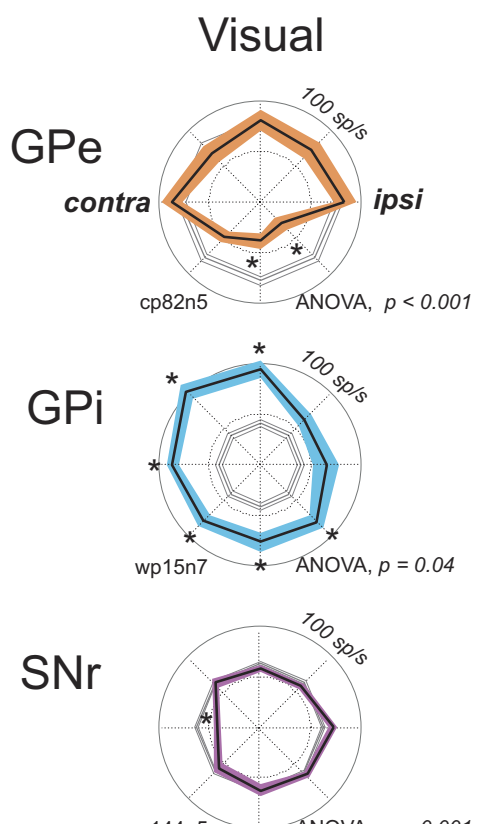

wp144n5 ANOVA, $p=0.001$
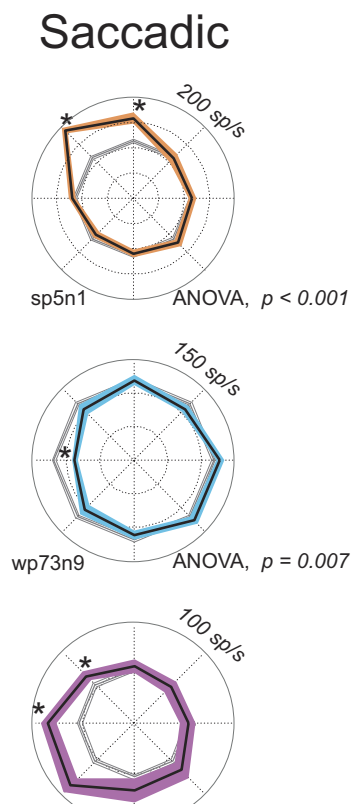

cp73n9 ANOVA, $p=0.02$
Reward
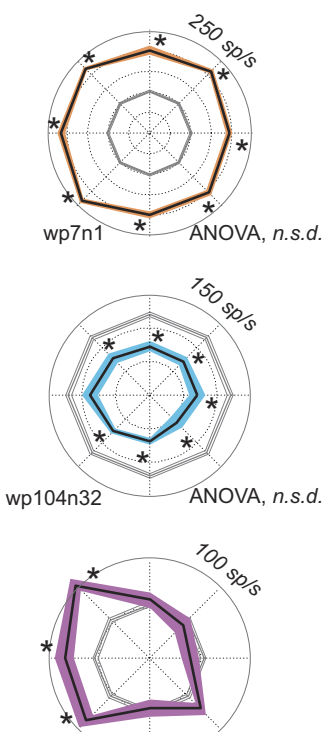

cp73n9 ANOVA, $p<0.001$
FIG. 7. Response field tuning curves from example neurons. Rows show data from neurons recorded in GPe (top, orange), GPi (middle, blue), and $\mathrm{SNr}$ (bottom, purple). Columns show tuning curves of the neurons' visual (left), saccade (middle), and reward (right) related signals. Firing rates during the respective signal epochs were plotted for the 8 target directions and connected by lines (means) and shaded areas ( \pm SEs). Gray lines show the baseline firing rates (mean \pm SE) for the neurons. The concentric circles depict 50 spikes/s increments, with peak of the scale shown near the top right of each graph. Relative to the recorded neurons, contralateral (contra) is to the left, ipsilateral (ipsi) to the right. ANOVA results are shown near the bottom right of each graph and individually significant directions by $t$-test $(P<0.05$, Bonferroni corrected $)$ are labeled with asterisks. Individual neurons' identification numbers are shown at the bottom left of each graph.

creasing type: 10/35, 29\%; decreasing type: 1/13, $8 \%$ ). The general conclusion from Fig. 8 is that the spatial tuning of GPe neurons became less contralateralized and broader as the events in a trial proceeded from visual to saccade to reward.

GPi task-modulated neurons were also analyzed, but we were unable to collect full data on the tuning curves for most of those neurons, so the data are sparse $(n=17)$. We show only the histograms of number of modulated directions (Fig. 9). A tendency for broader tuning as trials progressed seemed to be present in our GPi sample.

For comparison, $\mathrm{SNr}$ neurons were analyzed in the same way $(n=36)$. On average, visual responses of $\mathrm{SNr}$ neurons were lateralized (ANOVAs: $P=0.01$ for increasing signals

Visual

A

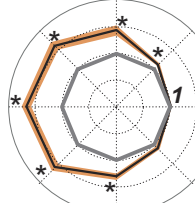

ANOVA, $p<0.00$
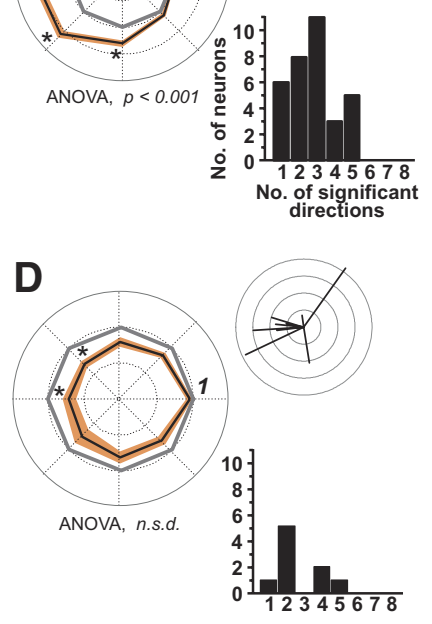

Saccadic

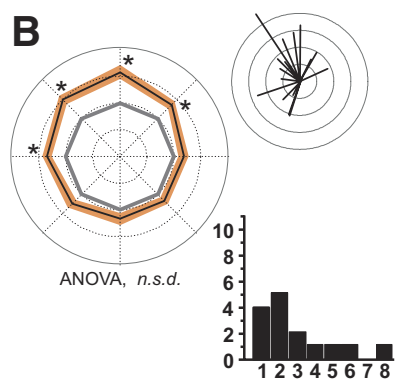

E

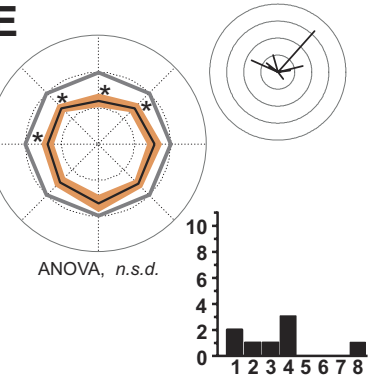

and $P=0.002$ for decreasing signals; Fig. 10, $A$ and $C$ ). These biases in the population were strongly contralateral (asterisks in Fig. 10, $A$ and $C$ ) due to underlying strong contralateral biases of individual neurons (top right insets). In those individual neurons, significant lateralized biases were found in $80 \%(8 / 10)$ of the increasing type and $75 \%(15 / 20)$ of the decreasing type. Saccade- and reward-related signals in our $\mathrm{SNr}$ neurons, however, did not show biased laterality (ANOVA, $P>0.05$ ).

As noted earlier, there seemed to be a broadening of response fields during a trial. To further analyze this, we quantitatively analyzed the shapes of the response fields for the GPe population. First, we considered the neurons with increasing

Reward
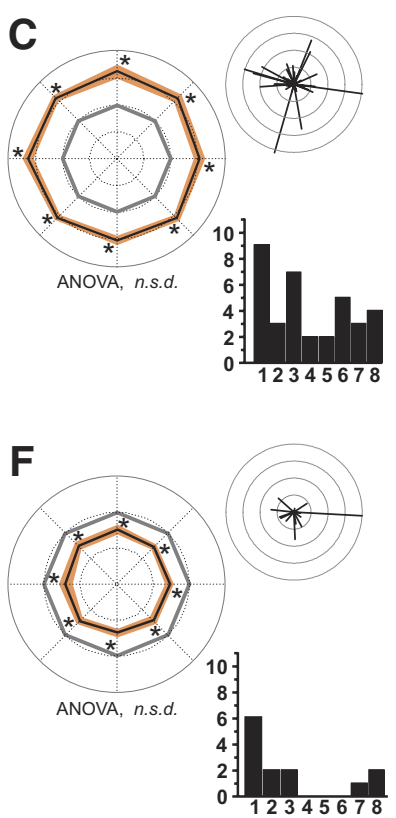

FIG. 8. Average response field tuning curves for the population of GPe neurons. Rows show data from neurons with increasing (top) or decreasing (bottom) signals relative to baseline. Columns show data from the visual (left), saccade (middle), and reward (right) related epochs. $A-F$ : in each panel, the average tuning curve (mean \pm $\mathrm{SE}$ ) of the sample of GPe neurons having the specific category of signal is shown at left. These average tuning curves were from normalized data in which each individual neurons' curve was scaled relative to its baseline firing rate (thus all baseline mean data $=1$ in these graphs). Smaller polar plots in top right insets represent the tuning curve average vectors (straight black lines) for each neuron that contributed to the average tuning curve (black lines). Histograms in bottom right insets show the number of significantly modulated directions found by $t$-test, which represents the sharpness of tuning (small numbers $=$ relatively narrow tuning; large numbers $=$ relatively broad tuning). Other conventions as in Fig. 7. For more details, see text. 


\section{Saccadic Reward}

\section{Increasing type}
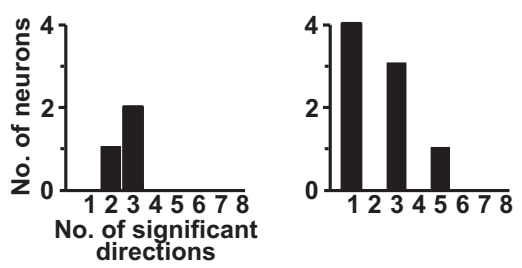

Decreasing type
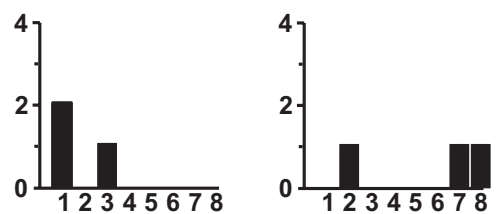

FIG. 9. Population results for GPi showing the number of significantly modulated directions (conventions as for the bottom right insets of Fig. 8). Visual category is absent because we found only one GPi neuron with a visual signal.

types of signals (Fig. 11). The method was described in detail elsewhere (Crapse and Sommer 2009). We normalized each individual response field to its maximal firing rate (Fig. 11A), which was set to one, and rotated the normalized curves so that direction of maximal firing rate was pointing to the left (Fig. $11 B$ ). With all of the tuning curves superimposed in this way, the firing rates and tuning directions were removed as factors, thus revealing their shapes. We computed average shapes from these individual curves (Fig. 11C, mean $\pm \mathrm{SE}$ ). Qualitatively, the shapes changed as we suspected from the previous analyses, becoming broader (enclosing more area) from visual (left) to saccadic (middle) to reward activity (right). This effect was quantified by calculating the areas within the tuning curves. The area calculations were performed on the normalized tuning

Visual

Saccadic
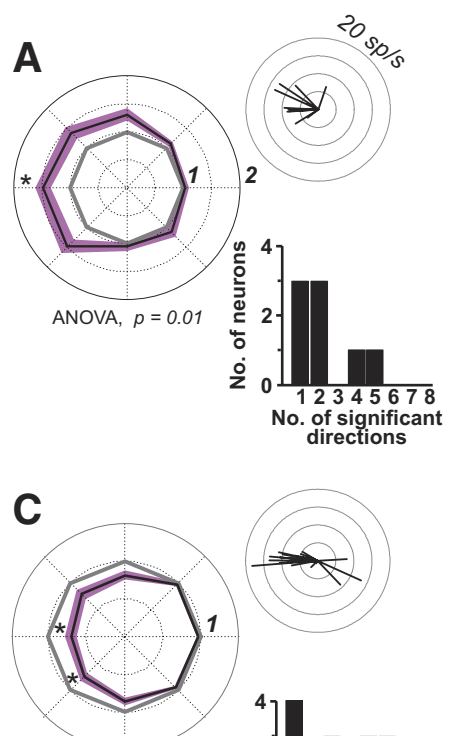

ANOVA, $p=0.002$

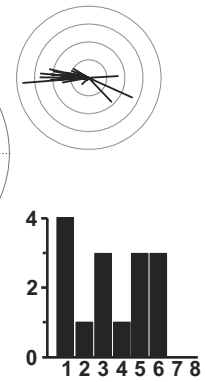

D

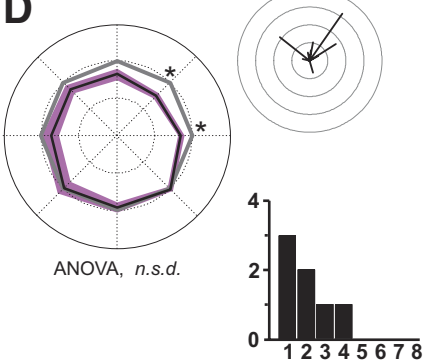

plots for each signal (from Fig. 11, $A$ or $B$ ). Using this absolute area value, the relative area was calculated as a percentage of the absolute area to the maximum possible area (i.e., the area of an equilateral octagon of radius one). This transformed the area data into a range for which $0 \%=$ narrowest possible tuning and $100 \%$ = broadest possible tuning. The distributions of relative areas are plotted in histograms in Fig. 11D. Although the relative areas of the saccadic tuning plots (mean $51 \%$ ) tended to be broader than the relative areas of the visual tuning plots (mean 46\%), the two were not significantly different from each other. However, both were significantly less than the relative areas of the reward tuning plots (mean 63\%, $t$-test).

Similar analyses were performed on decreasing types of signals (Fig. 12), except that the individual neuron firing rates were normalized to the trough of the tuning curve, which was set to 0.2 (innermost of the concentric circles). Relative areas of each tuning plot were calculated by dividing the absolute area values by the minimum area (i.e., equilateral octagon with radius 0.2 ). This value was always $>1$. To achieve a comparable breadth index as used in Fig. 11, we took the reciprocal of the relative area (always <1) and multiplied it by $100 \%$. Thus analogous to the result in Fig. 11, indices near 0\% represent extremely narrow tuning (a notch at the preferred direction and high firing rates elsewhere) and indices near $100 \%$ extremely broad tuning (similar decreases for all directions). The distributions of breadth indices are shown in Fig. $12 D$. We found no significant differences in the tuning curve breadths as a function of signal type for these decreasing modulations ( $P>0.05$ for all comparisons).

The same analyses were performed on our $\mathrm{SNr}$ sample (Supplemental Figs. S3 and S4), but they did not reveal any significant differences in the breadths of tuning $(P>0.05$ for all comparisons). When the distributions of breadth indices for each group of $\mathrm{SNr}$ neurons were compared with the respective

\section{Reward}
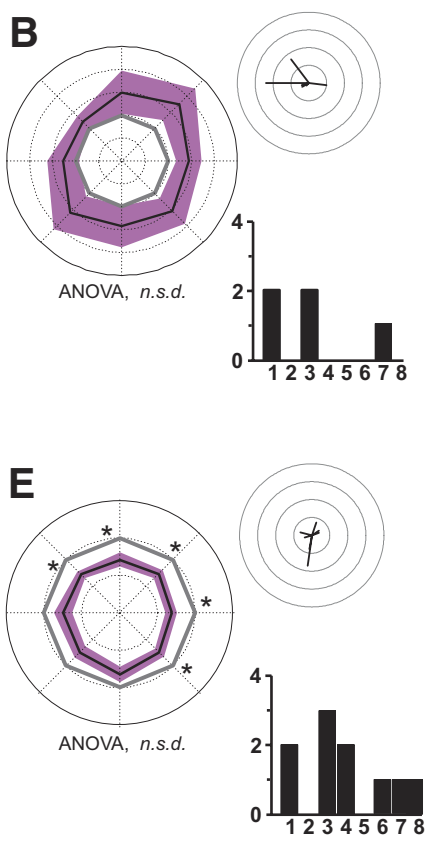

FIG. 10. Population tuning curves for $\mathrm{SNr}$ neurons. Conventions are the same as those in Fig. 8. Our SNr sample had no increasing type of saccaderelated neurons, so that category is absent. 


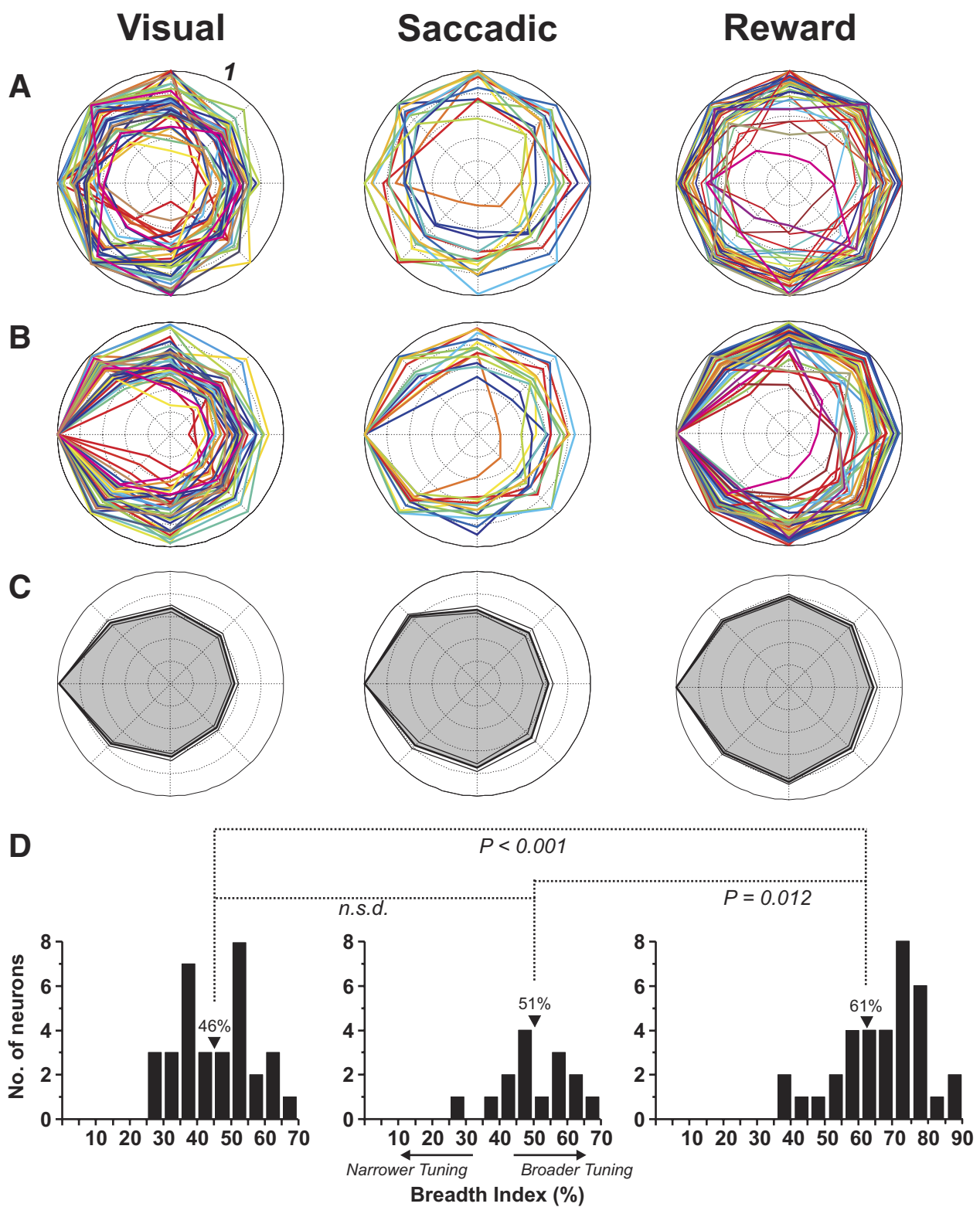

FIG. 11. Breadths of tuning curves for the increasing type of GPe neurons. For details of constructing the graphs, see text. Columns show data from the visual (left), saccade (middle), and reward (right) related epochs. A: tuning curves from individual GPe neurons with data normalized to peak firing rate (which is set to 1). Baseline data are irrelevant for these analyses and are not shown. $B$ : same tuning curves as in $A$, but rotated so that the peak firing rate for each neuron is to the left. $C$ : means and SEs of the normalized, rotated tuning curves from $B$. As suggested by the data in Fig. 8, $A-C$, the tuning curves did become more broadly tuned as trials progressed from visual to saccadic to reward events. $D$ : histograms showing the distribution of relative tuning curve areas for each signal. Tuning curve absolute areas were calculated as in $C$ for each neuron individually and then converted to relative areas by expressing them as a percentage of the maximal possible area (i.e., the area that would be enclosed by an equilateral octagon having all points $=1$ ). Arrowheads and labels show the mean of each distribution and the results of multiple $t$-tests are shown. data from GPe (e.g., increasing visual GPe vs. increasing visual $\mathrm{SNr}$, none of the comparisons was significantly different (t-test, $P>0.05)$.

\section{I S C U S S I O N}

Our findings support the hypothesis that GPe and GPi neurons are active in diverse ways during oculomotor behavior. The neurons have high firing rates that increase or decrease during visual, saccade, or reward events of a memory-guided task. Comparing between the structures, GPe neurons were strongly visual-related, whereas GPi neurons almost lacked visual activity. Reward activity was common in both structures but was found in a higher proportion in GPi than that in GPe. There was considerable saccade-related activity in both structures but infrequent delay activity. As expected from the GPe-to-SNr inhibitory projection, the signal content of GPe neurons closely resembled that of $\mathrm{SNr}$ neurons except for a general sign reversal. The signal contents of GPi and SNr had little in common. In both GPe and GPi, spatial response fields could be centered at nearly any amplitude and were relatively sharply tuned and contralaterally directed for visual signals, less distinctly tuned for saccadic signals, and largely omnidirectional for reward signals.

It is well established that control of eye movements involves the caudate nucleus and SNr (Basso and Liu 2007; Basso and Wurtz 2002; Handel and Glimcher 1999, 2000; Hikosaka and Wurtz 1983d,e, 1985; Hikosaka et al. 2000; Jiang et al. 2003; Liu and Basso 2008; Sato and Hikosaka 2002). Just because the caudate-SNr pathway is an important oculomotor circuit, however, does not mean that it is the only such circuit in the basal ganglia. When we began our study, the direct pathway through GPi and the indirect pathways through GPe had not been ruled out as oculomotor circuits; to our knowledge they simply had not been studied (with one exception: Kato and Hikosaka 1995). Traditionally, the GPi had been implicated in skeletomotor, not oculomotor, behavior. The GPe was known to be part of an indirect pathway that could influence the $\mathrm{SNr}$, 


\section{Visual}

A

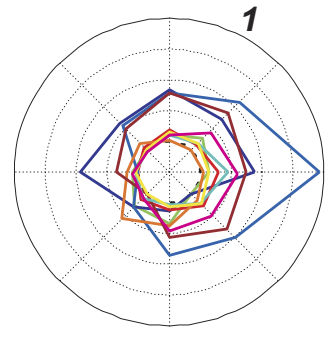

B

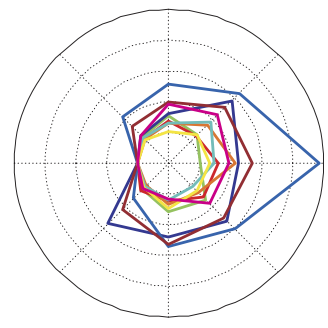

C

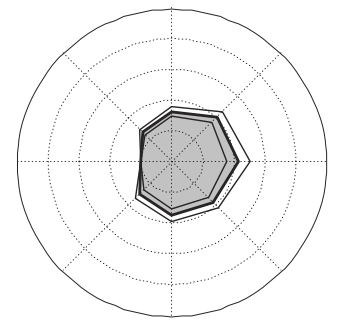

Saccadic
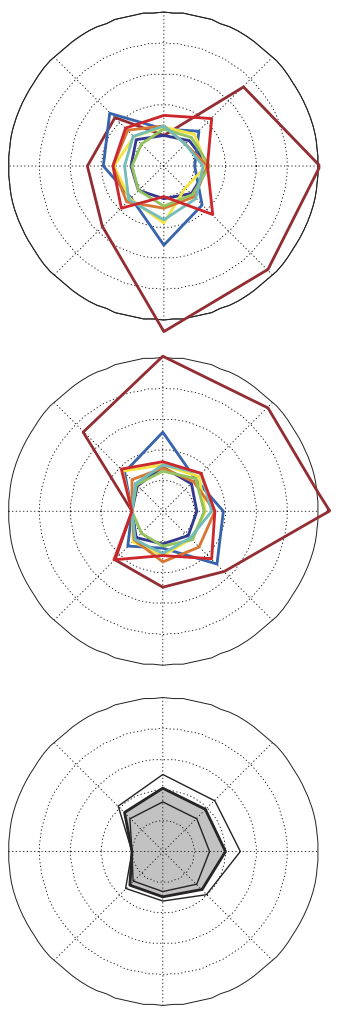

Reward
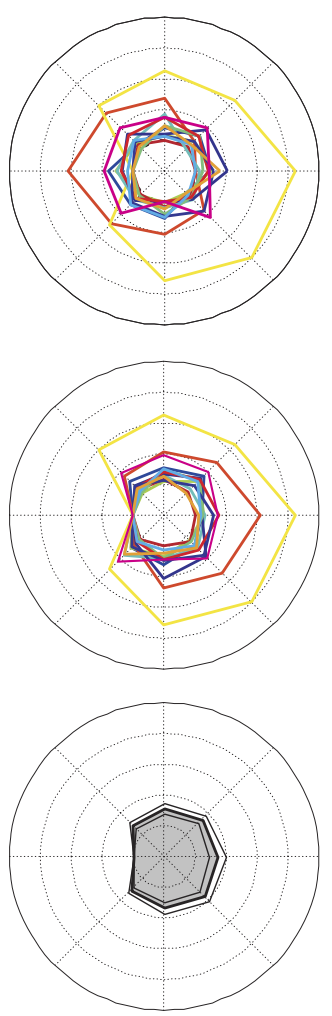

FIG. 12. Breadths of tuning curves for the decreasing type of GPe neurons. Conventions are the same as those in Fig. 11 except that each tuning curve is normalized to its minimum firing rate (set to 0.2 , innermost concentric ring) and thus the calculation of breadth index was different. For details of constructing the graphs and calculation of the index, see text.

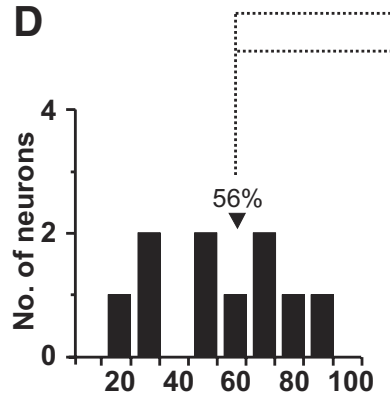

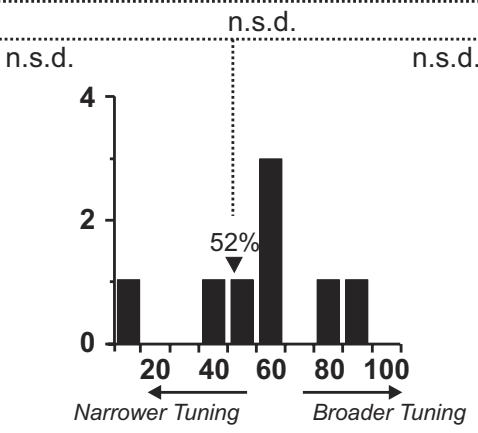

Breadth Index (\%)

but no quantified summaries of its signal content during oculomotor behavior had been reported. Very recently, accounts of pallidal signals during anti- and prosaccades (Yoshida and Tanaka 2009a) and smooth pursuit (Yoshida and Tanaka 2009b) have been published. Our data extend these new findings to provide a quantified assessment of the wide range of information carried by GPe and GPi neurons during saccadic behavior.

Our data reinforce the emerging view that the GPe and GPi may play an oculomotor role. Neurons in both structures (as in $\mathrm{SNr}$ ) have visual-, saccade-, and reward-related modulations. Similar signals are found in well-known oculomotor structures outside of the basal ganglia such as the superior colliculus (Goldberg and Wurtz 1972a,b; Schiller and Koerner 1971; Wurtz and Goldberg 1972a,b) and the frontal eye field (Bruce and Goldberg 1985; Schall 1991; Tehovnik et al. 2000). The signals are represented differently between the structures, as bursts and pauses relative to a high firing rate in $\mathrm{GPe}, \mathrm{GPi}$, and

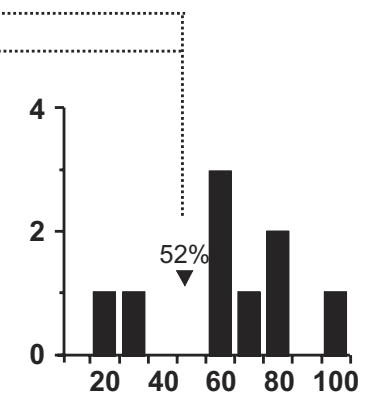

$\mathrm{SNr}$, but primarily as bursts relative to a low firing rate in superior colliculus, frontal eye field, and most other areas including the caudate nucleus, although the information carried by the signals in all of these structures is comparable. This is not to say that the signals from the various structures are equivalent in their behavioral impact. The GPi, in particular, seems to convey far fewer oculomotor signals than undisputed saccade-related structures such as the superior colliculus. Our point is that GPe and GPi neurons represent multiple events during oculomotor behavior using bursts and pauses as seen elsewhere in the visuosaccadic system, thus making them feasible candidates for participation in eye movements.

Three previous studies provided important data on the signals carried by GPe and GPi neurons during oculomotor behavior. The pioneering study of GPe by Kato and Hikosaka (1995) showed evidence for saccade-related activity in a task that also required lever presses. They used targets at multiple directions and eccentricities but did not quantify population 
response field characteristics. Hong and Hikosaka (2008) found that neurons in GPi carry visual signals representing negative rewards in a biased-reward saccade task. They used targets at $15^{\circ}$ eccentricity, left and right of fixation. Yoshida and Tanaka (2009a) reported saccade-related activity in GP using targets at $16^{\circ}$ eccentricity, in multiple directions. The eccentricities in the two recent studies (Hong and Hikosaka 2008; Yoshida and Tanaka 2009a) were close to the average eccentricity of response field centers that we reported $\left(18^{\circ}\right)$, but our mapping revealed that the range of optimal eccentricities was expansive $\left(2-64^{\circ}\right)$. Only $35 \%$ of our GP neurons had response field centers between 10 and $25^{\circ}$ eccentricity. It seems unlikely that the other $65 \%$ of neurons in our sample would have been adequately characterized in the prior work. Our systematic response field mapping may also explain why most fields in the present study were found to be spatially tuned, compared with an apparent prevalence of omnidirectional fields in the previous work.

A somewhat surprising difference between previous work (Hong and Hikosaka 2008; Kato and Hikosaka 1995; Yoshida and Tanaka 2009a,b) and our study pertains to saccade-related activity in GP, which was more prevalent in their data than in ours. This could be due to the subpopulation of GP neurons recorded. Neurons in the previous studies had relatively low baseline firing rates $(\sim 33-45$ spikes/s) characteristic of GP "border neurons" (DeLong 1971), whereas our neurons had higher firing rates $(\sim 80$ spikes/s) and seemed to be located throughout GPe and GPi (Supplemental Fig. 1A). In terms of anterior-posterior location, we estimated from MRI, atlases, and physiological landmarks that our pallidal neurons were clustered 4-7 $\mathrm{mm}$ posterior to the anterior commissure, but Yoshida and Tanaka (2009a) localized their sample to within 2 $\mathrm{mm}$ of the anterior commissure. Also, there were methodological differences between the studies. Yoshida and Tanaka (2009a) used memory-guided saccades, antisaccades, and prosaccades and found the highest saccade-related modulations for antisaccades. We did not test antisaccades, so we may have missed some potential saccade-related modulations. Yoshida and Tanaka (2009a) used a relatively broad epoch for defining saccade-related activity $(100 \mathrm{~ms}$ before to $200 \mathrm{~ms}$ after saccade initiation) and only required it to differ from delay activity. We analyzed a more restricted epoch, $50 \mathrm{~ms}$ before to $50 \mathrm{~ms}$ after saccade initiation, and required it to differ from both baseline and delay activity, to minimize false positives.

Neuronal recording results are correlational and do not demonstrate functional impact. More experiments in the future should attempt causal perturbations of GPe and GPi to see whether stimulation or inactivation affects oculomotor behavior. The latter manipulation was done in GPe by Yoshida and Tanaka (2009a) who found impairment in antisaccades. Some clinical and neurosurgical evidences suggest a causal oculomotor function for GPi. Deep brain stimulation of posteroventral GPi in human Parkinson's and Huntington's patients and posteroventral pallidotomy in Parkinson's patients have been shown to influence eye movements (Blekher et al. 2000; Fawcett et al. 2005; O'Sullivan et al. 2003; Straube et al. 1998).

Although we found neuronal signals related to vision, saccades, and rewards, some of the activity modulations may have been correlated with events that we did not monitor directly. One example could be the subtle contraction of neck muscles.
This is difficult to rule out even when recording from the most well understood oculomotor structures. Recently it was shown that the activity of saccade-related neurons in the superior colliculus and the frontal eye field is correlated with neck muscle activation (Elsley et al. 2007; Rezvani and Corneil 2008). The activity of some GPi and GPe neurons may be similarly complex, but this possibility does not weaken our fundamental finding that signals exist in both structures to implicate them in oculomotor behavior. Some of our neurons had small-eccentricity response field centers; for example, 39\% had centers at $\leq 10^{\circ}$ or smaller eccentricity and $72 \%$ had centers at $\leq 20^{\circ}$ eccentricity. In head unrestrained monkeys, saccades of $\leq 20^{\circ}$ amplitude are accompanied by only negligible head movement (reviewed by Freedman 2008). These data provide evidence, albeit indirect, that most of the movement-related activity modulation of our neurons was due to the saccades that were made rather than head movements that may have been attempted.

We found that GPe and GPi neurons had different distributions of signals. GPe neurons had more visual activity and GPi neurons had more reward-related activity. The high proportion of reward-related activity in GPi concurs with its connectivity. The GPi has reciprocal connections with substantia nigra pars compacta (Charara and Parent 1994; Smith et al. 1989) and sends efferents to the lateral habenula (Hazrati and Parent 1991; Lecourtier and Kelly 2007; Parent et al. 2001). Matsumoto and Hikosaka (2007) reported that the lateral habenula predicts negative reward value and provides inputs to compacta neurons that predict positive reward value. A follow-up study using antidromic stimulation to identify GPi projection neurons provided direct evidence that they contribute to the negative value signals in lateral habenula (Hong and Hikosaka 2008).

We found that in GPe neurons, too, reward-related signals were present. GPe neurons have no known direct connection with the habenula; instead, they interconnect mostly with other structures in the basal ganglia (Parent and Hazrati 1995). Many such structures, including $\mathrm{SNr}, \mathrm{GPi}$, and caudate nucleus, are implicated in reward processing, so the reward-related neurons in GPe might contribute to oculomotor behavior through them.

A prominent feature of GPe neurons was their strong visual responsivity. This characteristic hints at where the neurons may project. Two major targets of GPe are the subthalamic nucleus and $\mathrm{SNr}$ (Sato et al. 2000), structures that have many neurons with visual responses (Hikosaka and Wurtz 1983a,b,c,d; Hikosaka et al. 2000; Matsumura et al. 1992). Another major target of GPe neurons is GPi (Sato et al. 2000), but as we have shown, GPi neurons have little visual responsivity. The simplest explanation is that the GPe neurons that we studied preferentially project to subthalamic nucleus and $\mathrm{SNr}$, rather than to GPi. GPe neurons are inhibitory and, supporting the idea that our GPe neurons may influence the $\mathrm{SNr}$, we found that the valences of signals in our GPe and $\mathrm{SNr}$ samples were generally opposite; increasing signals predominated in the GPe former and decreasing signals in the $\mathrm{SNr}$.

In our analysis of the response fields, we identified a surprising trend: the fields became less lateralized and more omnidirectional as events proceeded from visual stimulation to saccade generation to reward delivery. The most striking difference (and the only change that was statistically significant) was the sudden broadening of fields at the final, reward delivery, stage of the task. Fundamentally, GPe and GPi 
neurons fired in advance of rewards regardless of the spatial details of a particular trial. The nonspatial nature of the activity reinforces the idea that it was related to predicting reward (a nonspatial entity) and was not a late response yoked to particular vectors of visual stimulation or saccadic movement.

Somewhat neglected in our report has been the potential role of the subthalamic nucleus, a glutamatergic basal ganglia structure (see Fig. 1A). We do not mean to minimize its potential importance. The connections between GPe and the subthalamic nucleus are reciprocal and much of the increasing type of modulation we see in the GPe could be due to excitatory inputs from the subthalamic nucleus. Future studies that use antidromic and orthodromic stimulation to identify more of the inputs and outputs of the GPe are needed to test hypotheses like these.

A provocative implication of our study is that both output nodes of the basal ganglia, the GPi and the SNr, may contribute to oculomotor behavior. We suggest that the functions of these two output nodes may complement each other. A major difference between the GPi and SNr is their anatomical realm of influence. The most prominent transthalamic targets of GPi are motor and premotor cortices (Alexander et al. 1986; Kayahara and Nakano 1996; Strick et al. 1995), whereas the SNr strongly targets the frontal eye field (Lynch et al. 1994). Thus the GPi may relay oculomotor information to parts of the cerebral cortex that the SNr does not reach. Subcortically, the GPi influences many brain stem structures but apparently not the superior colliculus, which is a well-known target of the SNr. It may be, then, that the GPi does not contribute to saccade generation per se. A more likely hypothesis is that it helps to integrate eye movements and skeletal movements. Taking into account its strong reward-related activity, the GPi may help to coordinate various modalities of action according to reward context.

\section{G R A N T S}

This work was supported by National Eye Institute Grant EY-017592 to M. A. Sommer.

\section{REFERENCES}

Alexander GE, DeLong MR, Strick PL. Parallel organization of functionally segregated circuits linking basal ganglia and cortex. Anпи Rev Neurosci 9: 357-381, 1986.

Anderson ME, Turner RS. A quantitative analysis of pallidal discharge during targeted reaching movement in the monkey. Exp Brain Res 86: 623-632, 1991.

Basso MA, Liu P. Context-dependent effects of substantia nigra stimulation on eye movements. J Neurophysiol 97: 4129-4142, 2007.

Basso MA, Wurtz RH. Neuronal activity in substantia nigra pars reticulata during target selection. J Neurosci 22: 1883-1894, 2002.

Blekher T, Siemers E, Abel LA, Yee RD. Eye movements in Parkinson's disease: before and after pallidotomy. Invest Ophthalmol Vis Sci 41: 2177 2183, 2000.

Bruce CJ, Goldberg ME. Primate frontal eye fields. I. Single neurons discharging before saccades. J Neurophysiol 53: 603-635, 1985.

Charara A, Parent A. Brainstem dopaminergic, cholinergic and serotoninergic afferents to the pallidum in the squirrel monkey. Brain Res 640: 155-170, 1994.

Crapse TB, Sommer MA. Frontal eye field neurons with spatial representations predicted by their subcortical input. J Neurosci 29: 5308-5318, 2009.

DeLong MR. Activity of pallidal neurons during movement. J Neurophysiol 34: 414-427, 1971.

DeLong MR, Georgopoulos AP. Motor functions of the basal ganglia. In: Handbook of Physiology. The Nervous System. Motor Control. Bethesda, MD: Am. Physiol. Soc. 1981, sect. 1, vol. II, pt. 2, p. 1017-1061.
Elias S, Joshua M, Goldberg JA, Heimer G, Arkadir D, Morris G, Bergman H. Statistical properties of pauses of the high-frequency discharge neurons in the external segment of the globus pallidus. J Neurosci 27: 2525-2538, 2007.

Elsley JK, Nagy B, Cushing SL, Corneil BD. Widespread presaccadic recruitment of neck muscles by stimulation of the primate frontal eye fields. J Neurophysiol 98: 1333-1354, 2007.

Fawcett AP, Moro E, Lang AE, Lozano AM, Hutchison WD. Pallidal deep brain stimulation influences both reflexive and voluntary saccades in Huntington's disease. Mov Disord 20: 371-377, 2005.

Freedman EG. Coordination of the eyes and head during visual orienting. Exp Brain Res 190: 369-387, 2008.

Goldberg ME, Wurtz RH. Activity of superior colliculus in behaving monkey. I. Visual receptive fields of single neurons. J Neurophysiol 35: 542-559, 1972a.

Goldberg ME, Wurtz RH. Activity of superior colliculus in behaving monkey. II. Effect of attention on neuronal responses. J Neurophysiol 35: 560-574, 1972b.

Graybiel AM, Ragsdale CW Jr. Fiber connections of the basal ganglia. Prog Brain Res 51: 237-283, 1979.

Handel A, Glimcher PW. Quantitative analysis of substantia nigra pars reticulata activity during a visually guided saccade task. J Neurophysiol 82: 3458-3475, 1999.

Handel A, Glimcher PW. Contextual modulation of substantia nigra pars reticulata neurons. J Neurophysiol 83: 3042-3048, 2000.

Hays AV, Richmond BJ, Optican BJ. A UNIX-based multiple process system for real-time data acquisition and control. WESCON Conf Proc 2: 1-10, 1982.

Hazrati LN, Parent A. Contralateral pallidothalamic and pallidotegmental projections in primates: an anterograde and retrograde labeling study. Brain Res 567: 212-223, 1991.

Hikosaka O, Sakamoto M, Usui S. Functional properties of monkey caudate neurons. I. Activities related to saccadic eye movements. J Neurophysiol 61: 780-798, 1989a.

Hikosaka O, Sakamoto M, Usui S. Functional properties of monkey caudate neurons. II. Visual and auditory responses. J Neurophysiol 61: 799-813, 1989 b.

Hikosaka O, Takikawa Y, Kawagoe R. Role of the basal ganglia in the control of purposive saccadic eye movements. Physiol Rev 80: 953-978, 2000.

Hikosaka O, Wurtz RH. Visual and oculomotor functions of monkey substantia nigra pars reticulata. I. Relation of visual and auditory responses to saccades. J Neurophysiol 49: 1230-1253, 1983a.

Hikosaka O, Wurtz RH. Visual and oculomotor functions of monkey substantia nigra pars reticulata. II. Visual responses related to fixation of gaze. J Neurophysiol 49: 1254-1267, 1983b.

Hikosaka O, Wurtz RH. Visual and oculomotor functions of monkey substantia nigra pars reticulata. III. Memory-contingent visual and saccade responses. J Neurophysiol 49: 1268-1284, 1983c.

Hikosaka O, Wurtz RH. Visual and oculomotor functions of monkey substantia nigra pars reticulata. IV. Relation of substantia nigra to superior colliculus. J Neurophysiol 49: 1285-1301, 1983d.

Hikosaka O, Wurtz RH. Effects on eye movements of a GABA agonist and antagonist injected into monkey superior colliculus. Brain Res 272: $368-$ $372,1983 \mathrm{e}$.

Hikosaka O, Wurtz RH. Modification of saccadic eye movements by GABArelated substances. II. Effects of muscimol in monkey substantia nigra pars reticulata. J Neurophysiol 53: 292-308, 1985.

Hikosaka O, Wurtz RH. The basal ganglia. Rev Oculomot Res 3: 257-281, 1989.

Hong S, Hikosaka $\mathbf{O}$. The globus pallidus sends reward-related signals to the lateral habenula. Neuron 60: 720-729, 2008.

Jiang H, Stein BE, McHaffie JG. Opposing basal ganglia processes shape midbrain visuomotor activity bilaterally. Nature 423: 982-986, 2003.

Judge SJ, Richmond BJ, Chu FC. Implantation of magnetic search coils for measurement of eye position: an improved method. Vision Res 20: 535-538, 1980.

Kato M, Hikosaka O. Function of the indirect pathway in the basal ganglia oculomotor system: visuo-oculomotor activities of external pallidum neurons. In: Age-Related Dopamine-Dependent Disorders (Monographs in Neural Sciences), edited by Segawa M, Nomura Y. Basel: Karger, 1995, p. 178-187. 
Kayahara T, Nakano K. Pallido-thalamo-motor cortical connections: an electron microscopic study in the macaque monkey. Brain Res 706: 337 342, 1996.

Lecourtier L, Kelly PH. A conductor hidden in the orchestra? Role of the habenular complex in monoamine transmission and cognition. Neurosci Biobehav Rev 31: 658-672, 2007.

Liu P, Basso MA. Substantia nigra stimulation influences monkey superior colliculus neuronal activity bilaterally. J Neurophysiol 100: 1098-1112, 2008.

Lynch JC, Hoover JE, Strick PL. Input to the primate frontal eye field from the substantia nigra, superior colliculus, and dentate nucleus demonstrated by transneuronal transport. Exp Brain Res 100: 181-186, 1994.

Martin RF, Bowden DM. Template Atlas of the Primate Brain. Seattle, WA: Primate Information Center, Univ. of Washington, 1996.

Matsumoto M, Hikosaka O. Lateral habenula as a source of negative reward signals in dopamine neurons. Nature 447: 1111-1115, 2007.

Matsumura M, Kojima J, Gardiner TW, Hikosaka O. Visual and oculomotor functions of monkey subthalamic nucleus. J Neurophysiol 67: 16151632, 1992.

Mays LE, Sparks DL. Dissociation of visual and saccade-related responses in superior colliculus neurons. J Neurophysiol 43: 207-232, 1980.

Mink JW. The basal ganglia: focused selection and inhibition of competing motor programs. Prog Neurobiol 50: 381-425, 1996.

Niijima K, Yoshida M. Electrophysiological evidence for branching nigral projections to pontine reticular formation, superior colliculus and thalamus. Brain Res 239: 279-282, 1982.

O'Sullivan JD, Maruff P, Tyler P, Peppard RF, McNeill P, Currie J. Unilateral pallidotomy for Parkinson's disease disrupts ocular fixation. J Clin Neurosci 10: 181-185, 2003.

Parent A, Hazrati LN. Functional anatomy of the basal ganglia. II. The place of subthalamic nucleus and external pallidum in basal ganglia circuitry. Brain Res Brain Res Rev 20: 128-154, 1995.

Parent M, Levesque M, Parent A. Two types of projection neurons in the internal pallidum of primates: single-axon tracing and three-dimensional reconstruction. J Comp Neurol 439: 162-175, 2001.

Paxinos G, Huang XF, Toga AW. The Rhesus Monkey Brain in Stereotaxic Coordinates. San Diego, CA: Academic Press, 2000.

Rezvani S, Corneil BD. Recruitment of a head turning synergy by lowfrequency activity in the primate superior colliculus. J Neurophysiol 100: 397-411, 2008.

Sato F, Lavallee P, Levesque M, Parent A. Single-axon tracing study of neurons of the external segment of the globus pallidus in primate. J Comp Neurol 417: 17-31, 2000
Sato M, Hikosaka O. Role of primate substantia nigra pars reticulata in reward-oriented saccadic eye movement. J Neurosci 22: 2363-2373, 2002.

Schall JD. Neuronal activity related to visually guided saccades in the frontal eye fields of rhesus monkeys: comparison with supplementary eye fields. J Neurophysiol 66: 559-579, 1991.

Schiller PH, Koerner F. Discharge characteristics of single units in superior colliculus of the alert rhesus monkey. J Neurophysiol 34: 920-936, 1971.

Smith Y, Lavoie B, Dumas J, Parent A. Evidence for a distinct nigropallidal dopaminergic projection in the squirrel monkey. Brain Res 482: 381-386, 1989

Soares J, Kliem MA, Betarbet R, Greenamyre JT, Yamamoto B, Wichmann $\mathbf{T}$. Role of external pallidal segment in primate parkinsonism: comparison of the effects of 1-methyl-4-phenyl-1,2,3,6-tetrahydropyridineinduced parkinsonism and lesions of the external pallidal segment. $J \mathrm{Neu}$ rosci 24: 6417-6426, 2004.

Sommer MA, Wurtz RH. Composition and topographic organization of signals sent from the frontal eye field to the superior colliculus. J Neurophysiol 83: 1979-2001, 2000

Sommer MA, Wurtz RH. What the brain stem tells the frontal cortex. I. Oculomotor signals sent from superior colliculus to frontal eye field via mediodorsal thalamus. J Neurophysiol 91: 1381-1402, 2004.

Straube A, Ditterich J, Oertel W, Kupsch A. Electrical stimulation of the posteroventral pallidum influences internally guided saccades in Parkinson's disease. J Neurol 245: 101-105, 1998.

Strick PL, Dum RP, Mushiake H. Basal ganglia "loops" with the cerebral cortex. In: Functions of the Cortico-Basal Ganglia Loop, edited by Kimura M, Graybiel AM. New York: Springer, 1995, p. 106-124.

Tehovnik EJ, Sommer MA, Chou IH, Slocum WM, Schiller PH. Eye fields in the frontal lobes of primates. Brain Res Brain Res Rev 32: 413-448, 2000.

White JM, Sparks DL, Stanford TR. Saccades to remembered target locations: an analysis of systematic and variable errors. Vision Res 34: 79-92, 1994.

Wurtz RH, Goldberg ME. Activity of superior colliculus in behaving monkey. III. Cells discharging before eye movements. J Neurophysiol 35: 575-586, 1972a.

Wurtz RH, Goldberg ME. Activity of superior colliculus in behaving monkey. IV. Effects of lesions on eye movements. J Neurophysiol 35: 587-596, 1972b.

Yoshida A, Tanaka M. Enhanced modulation of neuronal activity during antisaccades in the primate globus pallidus. Cereb Cortex 19: 206-217, 2009a.

Yoshida A, Tanaka M. Neuronal activity in the primate globus pallidus during smooth pursuit eye movements. Neuroreport 20: 121-125, 2009b. 